\title{
Anti-inflammatory effects of Simvastatin in patients with acute intracerebral hemorrhage in an intensive care unit
}

\author{
XIURONG ZHOU ${ }^{1}$, JIAFENG CHEN ${ }^{2,3}$, CHENGDONG WANG $^{4}$ and LILI WU ${ }^{2}$ \\ Departments of ${ }^{1}$ Neurosurgery and ${ }^{2}$ Neurology, People's Hospital, Weifang, Shandong 261041; \\ ${ }^{3}$ Intensive Care Unit, People's Hospital of Changle County, Weifang, Shandong 262400; \\ ${ }^{4}$ Central Laboratory, People's Hospital, Weifang, Shandong 261041, P.R. China
}

Received June 25, 2016; Accepted April 28, 2017

DOI: $10.3892 / \mathrm{etm} .2017 .5309$

\begin{abstract}
Intracerebral hemorrhage is one of the most common types of cerebrovascular disease in humans and often causes paralysis, a vegetative state and even death. Patients with acute intracerebral hemorrhage are frequently monitored in intensive care units (ICUs). Spontaneous intracerebral hemorrhage is associated with a higher rate of mortality and morbidity than other intracephalic diseases. The expression levels of inflammatory factors have important roles in inflammatory responses indicative of changes in a patient's condition and are therefore important in the monitoring and treatment of affected patients at the ICU as well as the development of therapeutic strategies for acute cerebral hemorrhage. The present study investigated the anti-inflammatory effects of Simvastatin in patients with acute intracerebral hemorrhage at an ICU, and inflammatory factors and cellular changes were systematically analyzed. The plasma concentrations of inflammatory factors, including interleukin (IL)-4, IL-6, IL-8 and IL-10, were evaluated by ELISAs. The plasma concentrations of inflammatory cellular changes were detected by using flow cytometry. The results demonstrated that after Simvastatin treatment of patients with acute cerebral hemorrhage at the ICU, the plasma concentrations of IL-4, IL-6, IL-8 and IL-10 were downregulated compared with those in placebo-treated controls. In addition, Simvastatin treatment at the ICU decreased lymphocytes, granulocytes and mononuclear cells in patients with acute cerebral hemorrhage. The levels of inflammatory factors were associated with brain edema in patients with acute cerebral hemorrhage treated at the ICU. In addition, the amount of bleeding was reduced in parallel with the inflammatory cell plasma concentration of lymphocytes, granulocytes and mononuclear cells. Importantly, Simvastatin treatment produced beneficial outcomes by improving brain
\end{abstract}

Correspondence to: Professor Lili Wu, Department of Neurology, Weifang People's Hospital, 151 Guangwen Street, Kuiwen, Weifang, Shandong 261041, P.R. China

E-mail:wulilihospital@126.com

Key words: acute intracerebral hemorrhage, intensive care unit, inflammatory responses, Simvastatin edema and reducing the amount of bleeding. In conclusion, the present study demonstrated the efficacy of Simvastatin in treating acute intracerebral hemorrhage and evidenced the association between inflammatory responses and the progress of affected patients at the ICU, thereby providing insight for applying effective therapies for patients with acute intracerebral hemorrhage.

\section{Introduction}

Cerebral hemorrhage is the most common type of cerebrovascular disease in humans (accounting for 20-30\%) and the least treatable subtype of hemorrhagic stroke (mortality rate, $30-40 \%$ ) (1). The most common manifestations in the clinic are cerebral arteriosclerosis, hypertension and intracranial vascular malformations (2). Cerebral hemorrhage is often induced by exertion and emotions, and most patients show sudden onset during activity. Cerebral hemorrhage usually causes severe dysfunction of the cerebral nervous system and loss of social functioning, self-care ability and further increases the burden on the family of affected patients $(3,4)$. Subarachnoid hemorrhage is one of the most serious types of cerebral hemorrhage and usually leads to death, as it is a devastating cerebrovascular disease with bleeding into the subarachnoid space $(5,6)$. While oral administration of anti-coagulants and surgical resection are the mainstay of cerebral hemorrhage treatment in the clinic, no effective therapeutic schedule is currently available to improve functional outcomes in patients with cerebral hemorrhage, particularly subarachnoid hemorrhage (7-9). Therefore, the development of therapeutic agents targeting cerebral hemorrhage is urgently required and the underlying molecular mechanisms require further elucidation in order to provide novel targets for identifying novel treatment approaches of human cerebrovascular diseases.

Patients with acute intracerebral hemorrhage are frequently monitored in intensive care units (ICUs) (10). Sudden intracerebral hemorrhage is associated with higher rates of mortality and morbidity than other intracephalic diseases (11). The expression levels of inflammatory factors have important roles in the inflammatory response associated with changes in a patient's condition and are important during monitoring and treatment at the ICU as well as for the development of 
therapeutic strategies for acute cerebral hemorrhage (12). In the majority of patients with cerebral hemorrhage, the condition triggers an immune activation sufficient to induce systemic inflammatory response syndrome (13). Inflammatory response has been reported to be associated with extra-cerebral organ dysfunction as well as delayed cerebral ischemia and the amount of bleeding, which is associated with poor outcome for patients with cerebral hemorrhage at an ICU (14). Leukocytosis has long been associated with adverse events after acute intracerebral hemorrhage (15). Therefore, controlling and monitoring inflammatory responses is essential for patients with acute intracerebral hemorrhage at the ICU.

As is known, the beneficial effects of statin drugs in reducing cardiovascular diseases have been predominantly attributed to their lipid-lowering effects (16). Recent studies suggested that the beneficial effects of statins are associated with their anti-inflammatory properties $(17,18)$. In addition, statins have important roles in changes in endothelial dysfunction, stabilizing the plaque and immune system regulation as well as anti-oxidant effects (19). Simvastatin is a statin drug that produces beneficial outcomes due to its anti-neoplastic effects and overcomes the resistance to serum withdrawal-induced apoptosis of lymphocytes from Alzheimer's disease patients (20). Numerous studies have investigated the anti-inflammatory effects of Simvastatin in different types of human diseases, such as cancer, chronic heart failure, diabetes and traumatic brain injury (21-24).

Furthermore, Simvastatin inhibited the aggregation of amyloid- $\beta$ in extracellular cortical and hippocampal plaques in cerebral hemorrhage, which is a widely accepted mechanism of action of cerebral hemorrhage pathology inhibitors and associated with the lowering of brain cholesterol levels in patients with cerebral hemorrhage $(25,26)$.

The present study investigated the anti-inflammatory effects of Simvastatin administered to patients after cerebral hemorrhage and hypothesized that it may protect neurons by regulating the inflammatory response. The anti-inflammatory properties of Simvastatin, including its capacity to inhibit inflammatory factor expression, decrease the inflammatory cellular plasma concentration of lymphocytes, granulocytes and mononuclear cells, and improve brain edema as well as reduce the amount of bleeding, were studied in patients with cerebral hemorrhage at the ICU. The results demonstrated that Simvastatin treatment led to the decrease of the levels of inflammatory factors, including interleukin (IL)-4, IL-6, IL-8 and IL-10. Furthermore, inflammatory factor levels were found to be associated with brain edema in patients with acute cerebral hemorrhage. The amount of bleeding was significantly depended on the inflammatory cellular plasma concentration of lymphocytes, granulocytes and mononuclear cells. Importantly, Simvastatin treatment produced beneficial outcomes with regard to improving brain edema and the amount of bleeding. However, further evaluation of additional clinical data is essential to fully elucidate the efficacy and tolerability of Simvastatin.

\section{Materials and methods}

Ethical approval and patient consent. This study was approved by the Ethics Committee of the People's Hospital of Changle
County (ref no. 10/CC06/124; Weifang, China). The phase-I study was performed from February 2006 to June 2012 according to the Guide for Chinese Clinical Experiments of Weifang People's Hospital (Weifang, China). The study was also performed in accordance with the European Medicines Agency requirements. All patients provided written informed consent prior to undergoing any procedures associated with the study.

Patients. A total of 146 patients who presented with intracerebral hemorrhage at the ICU were randomized into two groups, which were treated with Simvastatin or placebo, respectively. The inclusion criteria were patients with no heart disease or previous history of intracerebral hemorrhage. At baseline, patient age, body mass index and time since epilepsy diagnosis were similar between the two groups. In total, 101 patients who completed the maintenance period of the phase I study post-surgery (minimal invasive puncture and drainage vs. endoscopic surgery) were included in the analysis of the therapeutic effects of Simvastatin. Patients received Simvastatin at dosages of $0.08,0.16,0.24,0.30$ and $0.36 \mathrm{mg} / \mathrm{kg}$ based on a previous clinical trial (26).

${ }^{18} \mathrm{~F}$-Fluorodeoxyglucose positron emission tomography imaging (FDG-PET). FDG-PET was used to analyze brain edema and the amount of bleeding by using statistical parametric mapping (SPM) software (SPM, version 2; Wellcome Department of Imaging Neuroscience, University College London, London, UK). FDG-PET images were spatially normalized onto the Montreal Neurological Institute (MNI) PET brain template (MNI, McGill University, Montreal, QC, Canada), which defined regions of interest. Normalized images were smoothed by convolution with a $10-\mathrm{mm}$ full width at half maximum Gaussian kernel to increase the signal-to-noise ratio. Detailed procedures for FDG-PET acquisition and image processing were described in a previous study (27).

Vasospasm analysis. In the present clinical study, vasospasm in patients with intracerebral hemorrhage was defined using clinical and angiographic criteria for vasospasm. Symptomatic vasospasm was diagnosed and recorded as abnormal neurological status as described in a previous study (28).

ELISA. The levels of IL-4 (cat no. D4050), IL6 (cat no. D6050), IL-8 (cat no. D8000C) and IL-10 (cat no. DY417) (all from R\&D Systems, Inc., Minneapolis, MN, USA) in the peripheral blood of patients with intracerebral hemorrhage was assessed by using commercialized human interleukin ELISA kits. The ELISAs were performed according to the manufacturer's instructions. The results were measured at $450 \mathrm{~nm}$ with an ELISA reader and finally converted to concentrations of IL-4, IL6, IL-8 and IL-10.

Modified neurological severity score (MNSS) analysis. The patients with intracerebral hemorrhage at the ICU were subjected to MNSS assessment at 7, 14, 21, 28, 35 and 42 days of treatment ( $n=26$ in Simvastatin and $n=18$ in placebo group). MNSS analysis comprised sensory, motor, reflex and balance experiments. A scale of 0-18 was used to grade neurological function (normal score, 0; maximal deficit score, 18). The 
higher the score, the higher was the severity of injury in patients with intracerebral hemorrhage at the ICU.

Behavioral assessment. Behavioral assessment was performed on post-operative days 7, 14, 21 and 28, 35 and 42 for intracerebral hemorrhage patients. The assessment parameters, including left limb movement and coordination of movement, were evaluated using the modified Tarlov scores as follows: Severe level, possible limb movement and partial limb paralysis (1-4 points); moderate level, failure to jump and stand normally (4-7 points); primary level, failure to stand while being capable of joint movement (7-9 points); normal function (9-10 points). According to the study design, the assessment and Tarlov scoring were performed for each patient at the ICU independently and then averaged.

Efficacy and safety assessment. Efficacy assessment included determination of the maximum tolerated dose (MTD) in cerebral hemorrhage patients and dose-limiting toxicity in the presence of Simvastatin. Safety assessments included the incidence rates $(\geq 10 \%)$ of the most frequent treatment-associated adverse events in a 42-day treatment period in the drug treatment groups. The efficacy and safety data included all patients with cerebral hemorrhage receiving Simvastatin.

Flow cytometry. Peripheral blood was drawn from patients with cerebral hemorrhage and total leukocytes were extracted using a Human Leukocyte Extraction kit (Invitrogen; Thermo Fisher Scientific, Inc., Waltham, MA, USA). Inflammatory cellular plasma concentrations of lymphocytes, granulocytes and mononuclear cells were analyzed by flow cytometry (BD FACSDiva $^{\mathrm{TM}}$ v. 6.1.3 software; BD Biosciences, San Jose, CA, USA) as previously described (29).

Statistical analysis. Statistical analysis was performed by using SPSS 19.0 software (IBM Corp., Armonk, NY, USA) and Excel (2010 version; Microsoft Corporation, Redmond, WA, USA). Values are expressed as the mean \pm standard error of the mean. Statistical tests for data analysis included Fisher's exact test, log-rank test, Chi-square test, and Student's 2-tailed $\mathrm{t}$-test. $\mathrm{P}<0.05$ was considered to indicate a statistically significant difference.

\section{Results}

Characteristics of patients with cerebral hemorrhage. A total of 146 patients with cerebral hemorrhage who were candidates for intravenous injection were included in the present clinical study. The mean age of the patients was 47 years. All patients were randomized into two groups and treated with Simvastatin $(n=88)$ or placebo $(n=58)$ by intravenous injection. The number of male patients with cerebral hemorrhage $(n=94,64.4 \%)$ was slightly higher than that of female patients $(n=52,35.6 \%)$. The characteristics of patients with cerebral hemorrhage are summarized in Table I. Furthermore, 101 (69\%) patients with cerebral hemorrhage continued to complete the maintenance period of the phase-I study.

Duration of treatment, dose-limiting toxicities and MTD. The median overall duration of Simvastatin treatment was
Table I. Characteristics of patients with intracerebral hemorrhage $(n=146)$.

\begin{tabular}{lc}
\hline Parameter & Value \\
\hline Gender & $94(64.4 \%)$ \\
Male & $52(35.6 \%)$ \\
Female & 47.4 (Range: $35.4-65.8)$ \\
Mean age (years) & \\
Drug therapy & $58(60.3 \%)$ \\
Simvastatin & $58(39.7 \%)$ \\
Placebo & $101(69.2 \%)$ \\
Trial participation & $35(30.8 \%)$ \\
Complete & \\
Incomplete & Plasma glucose (mmol/l) \\
Blood pressure (mmHg) & $142 \pm 6$ (normal range: $90-140)$ \\
\hline
\end{tabular}

Values are expressed as $\mathrm{n}(\%)$, range or mean \pm standard error of the mean.

four weeks for patients with cerebral hemorrhage at the ICU. Within the cohort, subgroups were treated with $0.08,0.16,0.24$, 0.30 and $0.36 \mathrm{mg} / \mathrm{kg}$ Simvastatin. As presented in Table II, $0.30 \mathrm{mg} / \mathrm{kg}$ Simvastatin once a day was identified as the MTD and $0.24 \mathrm{mg} / \mathrm{kg}$ of Simvastatin once a day was identified as dose-limiting toxicity. The group treated with the lowest dose of Simvastatin presented with the lowest number of adverse reactions. It was observed that the common treatment-associated adverse events of Simvastatin injection were hypertension, proteinuria, fatigue, diarrhea, vomiting, rash, constipation and peripheral edema. For most of the patients with cerebral hemorrhage, a reduction of the drug dose was required due to cumulative toxicity after treatment with the MTD. Therefore, most of the patients that were subsequently enrolled in the study were treated with Simvastatin at a dose of $0.20 \mathrm{mg} / \mathrm{kg}$ to ensure tolerability and therapeutic efficacy of Simvastatin. The most common grade $\geq 3$ adverse events in patients with cerebral hemorrhage according to Common Toxicity Criteria were hypertension and proteinuria (15\% each; Table III).

Efficacy of Simvastatin in patients with cerebral hemorrhage. The efficacy of Simvastatin treatment in patients with acute cerebral hemorrhage was assessed in this clinical study. Clinical examination demonstrated that compared with the placebo group, arthralgia and body pain were markedly improved in the drug treatment groups after 4 weeks of therapy regiment. Furthermore, the levels of the inflammatory factors IL-4, IL-6, IL-8 and IL-10 were decreased after treatment with Simvastatin in patients with acute cerebral hemorrhage in the ICU (Fig. 1). Furthermore, systemic inflammatory response syndrome was found to be associated with brain edema in the patients with acute cerebral hemorrhage. Simvastatin treatment improved the degree of brain edema and the amount of bleeding (Fig. 2). Of note, the results indicated that the inflammatory cellular plasma concentration of lymphocytes, granulocytes and mononuclear cells was recovered to normal levels after 
Table II. Adverse events (n) occurring during treatment with an overall incidence of $\geq 10 \%$.

\begin{tabular}{lcccc}
\hline & & \multicolumn{3}{c}{ Simvastatin $(\mathrm{mg} / \mathrm{kg})$} \\
\cline { 3 - 5 } Adverse event & Total $(\%) \mathrm{n}=40$ & $0.08-0.16(\%) \mathrm{n}=14$ & $0.24-0.30(\%) \mathrm{n}=16$ & $0.36(\%) \mathrm{n}=10$ \\
\hline Hypertension & $10(25)$ & $2(5)$ & $3(7.5)$ & $5(12.5)$ \\
Proteinuria & $8(20)$ & $2(5)$ & $2(5)$ & $4(10)$ \\
Fatigue & $4(10)$ & $1(2.5)$ & $1(2.5)$ & $2(5)$ \\
Diarrhea & $5(12.5)$ & $1(2.5)$ & $2(5)$ & $2(5)$ \\
Vomiting & $4(10)$ & $1(5)$ & $1(2.5)$ & $2(5)$ \\
Rash & $6(15)$ & $2(5)$ & $2(5)$ & $3(5)$ \\
Constipation & $7(17.5)$ & $2(5)$ & $3(7.5)$ & $3(7.5)$ \\
Peripheral edema & $8(20)$ & & & $2(5)$ \\
\hline
\end{tabular}

Table III. Treatment-associated hypertension and proteinuria graded by Common Toxicity Criteria ( $\geq 20 \%)$.

\begin{tabular}{|c|c|c|c|c|}
\hline \multirow[b]{2}{*}{ Adverse event/grade } & \multirow[b]{2}{*}{ Total $(\%) \mathrm{n}=40$} & \multicolumn{3}{|c|}{ Simvastatin (mg/kg) } \\
\hline & & $0.08-0.16(\%) \mathrm{n}=14$ & $0.24-0.30(\%) \mathrm{n}=16$ & $0.36(\%) \mathrm{n}=10$ \\
\hline \multicolumn{5}{|l|}{ Hypertension } \\
\hline 1 & $3(7.5)$ & $0(0)$ & $1(2.5)$ & $2(5)$ \\
\hline 2 & $3(7.5)$ & $1(2.5)$ & $1(2.5)$ & $1(2.5)$ \\
\hline 3 & $4(10)$ & $1(2.5)$ & $1(2.5)$ & $2(5)$ \\
\hline Total & $10(25)$ & $2(5)$ & $3(7.5)$ & $5(12.5)$ \\
\hline \multicolumn{5}{|l|}{ Proteinuria (\%) } \\
\hline $1(2.5)$ & $2(5)$ & $0(0)$ & $1(2.5)$ & $2(5)$ \\
\hline $2(5)$ & $3(7.5)$ & $1(2.5)$ & $0(0)$ & $1(2.5)$ \\
\hline $3(7.5)$ & $2(5)$ & $1(2.5)$ & $1(2.5)$ & $1(2.5)$ \\
\hline Total & $8(20)$ & $2(5)$ & $2(5)$ & $4(10)$ \\
\hline
\end{tabular}

A
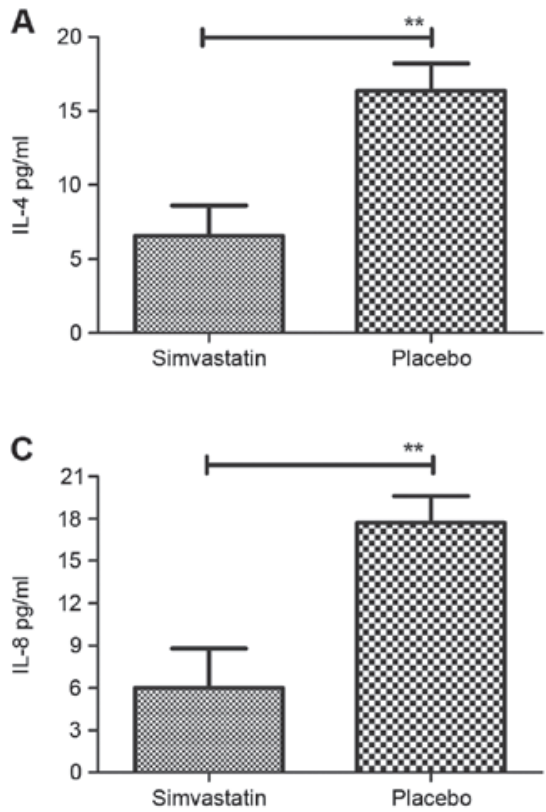
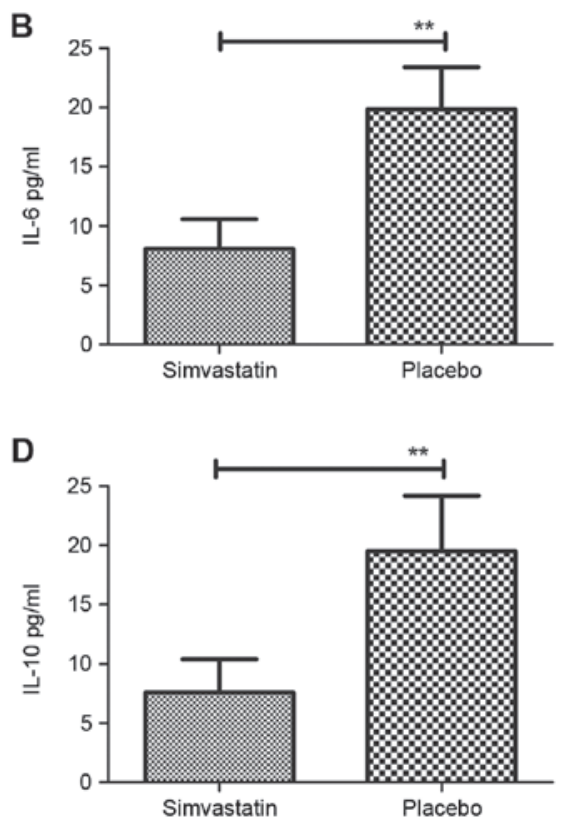

Figure 1. Analysis of the plasma concentration of inflammatory factors in patients with intracerebral hemorrhage treated with Simvastatin or placebo at the ICU. Plasma concentration of (A) IL-4, (B) IL-6, (C) IL-8 and (D) IL-10 in patients with intracerebral hemorrhage in ICU after a 42-day treatment period. Values are expressed as the mean \pm standard error of the mean determined from triplicate samples. ${ }^{* *} \mathrm{P}<0.01$, Simvastatin vs. placebo group. ICU, intensive care unit; IL, interleukin. 
A

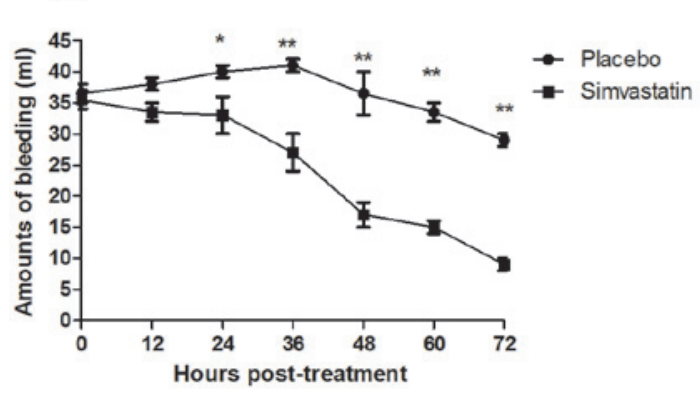

B

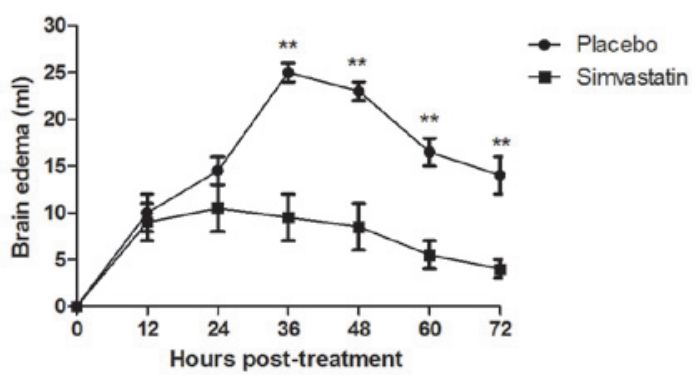

Figure 2 Analysis of brain edema and amount of bleeding in patients with intracerebral hemorrhage after treatment with Simvastatin or placebo at the ICU. (A) Brain edema was analyzed after a 42-day treatment period in patients with intracerebral hemorrhage at the ICU. (B) Amount of bleeding of patients with intracerebral hemorrhage at the ICU as determined by ${ }^{18} \mathrm{~F}$-fluorodeoxyglucose positron emission tomography imaging. Values are expressed as the mean \pm standard error of the mean determined from triplicate samples. ${ }^{*} \mathrm{P}<0.05$ and ${ }^{* *} \mathrm{P}<0.01$, Simvastatin vs. placebo group. ICU, intensive care unit.

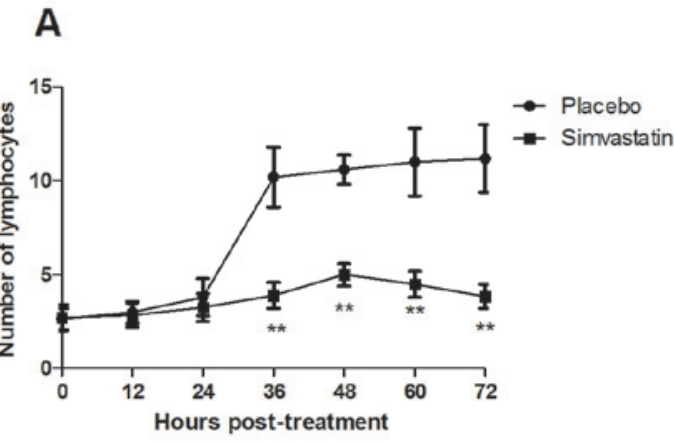

B

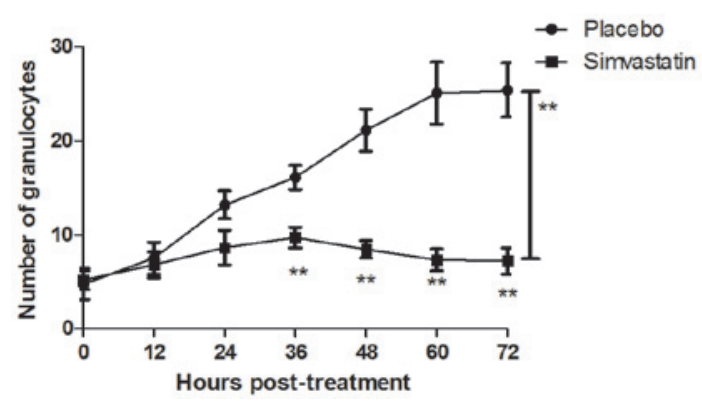

C

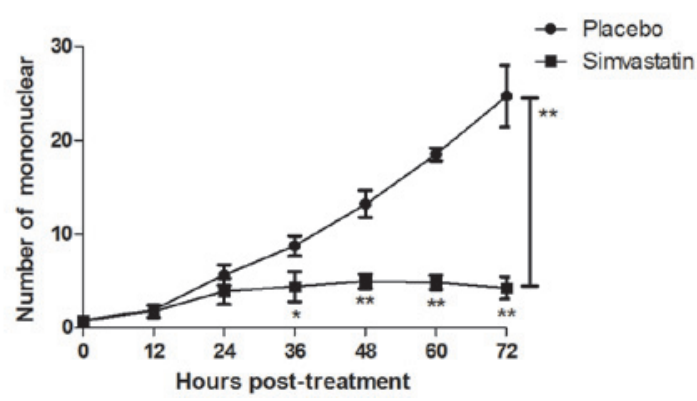

Figure 3. Analysis of leucocytes in plasma of patients with intracerebral hemorrhage after treatment with Simvastatin or placebo. (A-C) Inflammatory cellular plasma concentration of (A) lymphocytes, (B) granulocytes and (C) mononuclear cells was measured after treatment with Simvastatin or with placebo as a control. Values are expressed as the mean \pm standard error of the mean determined from triplicate samples. ${ }^{*} \mathrm{P}<0.05$ and ${ }^{* *} \mathrm{P}<0.01$, Simvastatin vs. placebo group.

treatment with Simvastatin (Fig. 3). Furthermore, the results revealed that Simvastatin treatment significantly improved the frequency of vasospasms in patients with acute cerebral hemorrhage compared with that in the placebo group (Fig. 4). Collectively, the results indicated that Simvastatin was efficient in treating acute intracerebral hemorrhage and that the inflammatory response was in parallel with the progress of patients with acute intracerebral hemorrhage in an ICU setting, which may provide insight for applying effective therapies for patients with acute intracerebral hemorrhage.

Simvastatin treatment improves the survival of patients with cerebral hemorrhage. In order to explore whether therapy with Simvastatin was effective for patients with cerebral hemorrhage in vivo, the recurrent activity of convulsion in cerebral

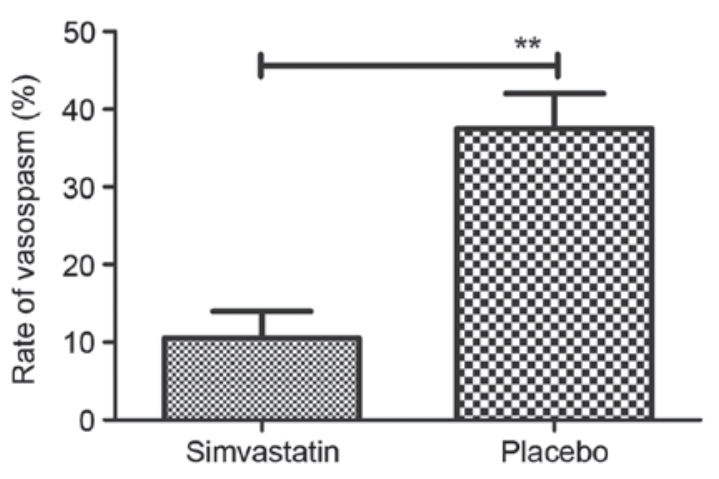

Figure 4. Rate of symptomatic vasospasms associated with an increasing inflammatory response burden on day 42 . Values are expressed as the mean \pm standard error of the mean determined from triplicate samples. ${ }^{* *} \mathrm{P}<0.01$, Simvastatin vs. placebo group. 
A

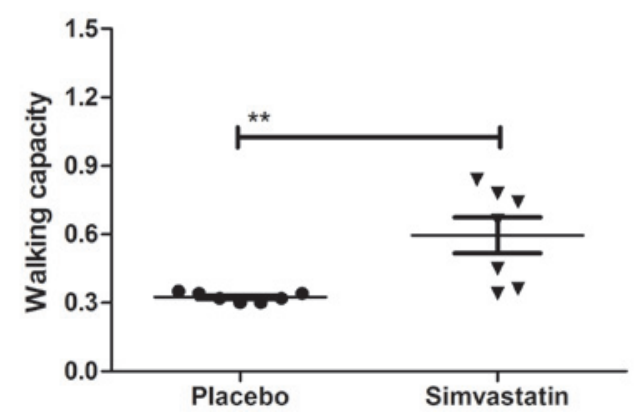

B

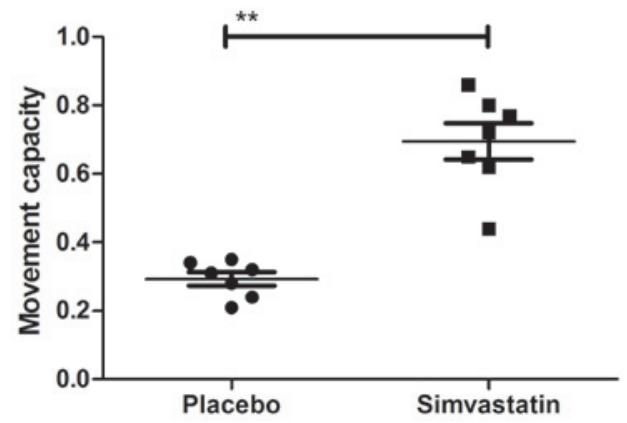

Figure 5. Walking and movement capacities of patients with intracerebral hemorrhage after treatment with Simvastatin or placebo. (A) Walking capacity on day 42. (B) Movement capacity on day 42. Values are expressed as the mean \pm standard error of the mean determined from triplicate samples. "* P<0.01, Simvastatin vs. placebo group.

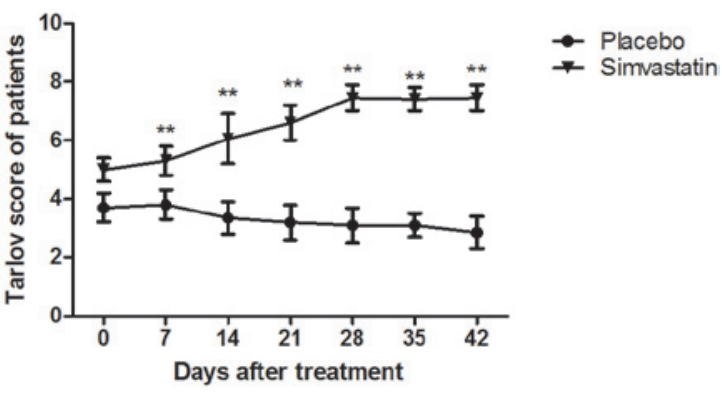

Figure 6. Behavioral assessment of patients with cerebral hemorrhage at the intensive care unit through evaluation of motor function. Behavioral assessment was performed by the modified Tarlov scale. The assessment parameters included left limb movement. Values are expressed as the mean \pm standard error of the mean determined from triplicate samples. ${ }^{* *} \mathrm{P}<0.01$, Simvastatin vs. placebo group.

hemorrhage patients was assessed. The results in Fig. 5 demonstrated that movement capacities, including limb coordination and walking, were significantly improved in Simvastatin-treated patients with hemorrhage lesions compared with those in patients treated with placebo $(\mathrm{P}<0.01)$. However, no significant changes in arterial blood pressure, body weight and body temperature were observed (data not shown). As presented in Fig. 6, the Tarlov scores revealed that the therapeutic effects of Simvastatin were significant in patients with cerebral hemorrhage compared with those in the placebo group $(\mathrm{P}<0.01)$. The MNSS test demonstrated that the Simvastatin-treated patients with cerebral hemorrhage exhibited significant functional improvement compared with the placebo group (Fig. 7). Furthermore, a long-term survival observation over a 1,664-day period after treatment with Simvastatin in patients with cerebral hemorrhage was performed. The results inFig. 8 revealed that the survival of patients was prolonged after treatment with Simvastatin. Taken together, the results suggested that Simvastatin was efficient in patients with cerebral hemorrhage in an ICU setting.

\section{Discussion}

Intracerebral hemorrhage is the most common type of cerebrovascular disease in humans and patients with acute intracerebral hemorrhage are frequently monitored in ICUs (30). Spontaneous

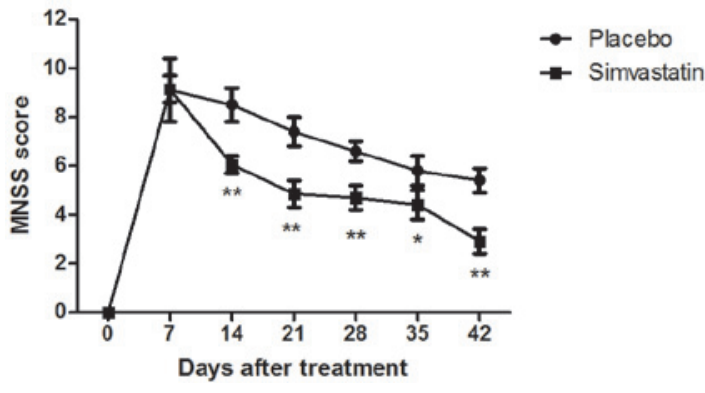

Figure 7. Treatment with Simvastatin promoted functional recovery, decreased brain water content and reduced the hemorrhage volume after intracerebral hemorrhage. The MNSS was determined to determine the therapeutic effects of Simvastatin from 1 to 42 days after intracerebral hemorrhage with placebo as control. Values are expressed as the mean \pm standard error of the mean determined from triplicate samples. ${ }^{*} \mathrm{P}<0.05$ and ${ }^{* *} \mathrm{P}<0.01$, Simvastatin vs. placebo group. MNSS, modified neurological severity score.

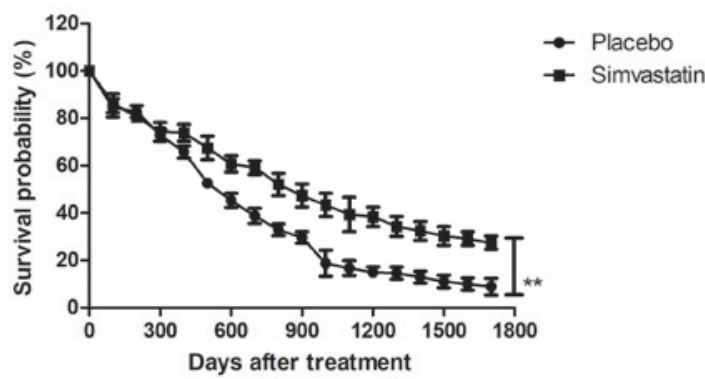

Figure 8 . Survival rates of patients with intracerebral hemorrhage over a 1,664-day survival period after Simvastatin or placebo treatment. Values are expressed as the mean \pm standard error of the mean determined from triplicate samples. ${ }^{* *} \mathrm{P}<0.01$, Simvastatin vs. placebo group.

and acute cerebral hemorrhage leads to high morbidity and mortality in ICUs worldwide $(31,32)$. It was previously indicated that systemic inflammatory response syndrome is associated with intracerebral hemorrhage (13). Simvastatin is an efficient drug that improves the neurological outcome after experimental intracerebral hemorrhage (33). In addition, Simvastatin was reported to reduce the inflammatory response in the treatment of various human diseases (34). The present study investigated the anti-inflammatory effects of Simvastatin in patients with 
acute intracerebral hemorrhage in an ICU. Recurrent bleeding after acute intracerebral hemorrhage is a major cause of morbidity and mortality in ICUs and a previous study suggested that the inflammatory response is associated with spontaneous intracerebral hemorrhage patients in an ICU setting (35). The present study revealed that Simvastatin treatment improved the inflammatory response and had beneficial effects in intracerebral hemorrhage patients in an ICU. Furthermore, numerous studies have shown that cerebral hemorrhage caused neuronal damage and further aggravated brain damage to even lead to the development of contralateral limb dysfunction (36-38). In cerebral hemorrhage, the blood often overflowed directly into the brain parenchyma. The clinical data of the present study demonstrated that contralateral limb dysfunction, frequency of vasospasm, degree of brain edema and the amount of bleeding were improved by treatment with Simvastatin once a day.

A previous study indicated that the possible mechanism underlying the dysfunction of the inflammatory response may be associated with leakage from small intracerebral arteries (39). Dysfunction of the inflammatory response may be an important pathophysiological factor in intracerebral hemorrhage and other human cerebrovascular diseases. A previous study has indicated that early inflammation contributes to edema after intracerebral hemorrhage in an ICU setting (40). A review on inflammation after intracerebral hemorrhage based on available evidences from preclinical and clinical studies suggested that inflammatory mechanisms are involved in the progression of intracerebral hemorrhage-induced secondary brain injury (41). In addition, the therapeutic benefit of anti-inflammatory and angiogenesis-inducing treatments in intracerebral hemorrhage has been investigated (42). The present study first assessed the anti-inflammatory effects of Simvastatin in patients with acute intracerebral hemorrhage in an ICU. The results revealed that IL-4, IL-6, IL-8 and IL-10 levels were downregulated by Simvastatin compared to those in the placebo group. Furthermore, in parallel with the decrease of inflammatory factors, the degree of brain edema and the amount of bleeding in patients with intracerebral hemorrhage was also reduced, along with an increased survival rate. These findings suggested that inhibition of inflammatory factors is beneficial for reducing the degree of brain injury and promoting functional recovery (43).

In the present study, Simvastatin was evaluated as a therapeutic agent for treating patients with intracerebral hemorrhage. In the majority of cases, cerebral hemorrhage is non-traumatic and caused by rupture of vessels in the brain parenchyma (44). The findings of the present study were consistent with those of previous studies in terms of Simvastatin exerting beneficial effects by inhibiting the expression of inflammatory factors in clinical trials $(45,46)$. The number of detection methods used for measuring the disease progression of intracranial hemorrhage has been expanding to include imaging cerebral physiology, PET, computed tomography and magnetic resonance imaging (47). In addition, numerous molecular markers were found to be associated with cerebral hemorrhage, which may support its diagnosis and determination of its extent, and may further be utilized for treating hemorrhage via target cells associated with hemostasis $(48,49)$. However, in terms of the overall survival rate of patients, further development or combined therapies are required for achieving better outcomes for patients with intracerebral hemorrhage in an ICU setting.
Of note, the present and previous studies indicated that Simvastatin is a prospective candidate drug for clinical therapy of patients with cerebrovascular disease $(50,51)$.

In conclusion, the present study proved the efficacy of Simvastatin in treating acute intracerebral hemorrhage and indicated that the inflammatory response was in parallel with the progression of patients with acute intracerebral hemorrhage in an ICU setting, which may provide insight for applying effective therapies for patients with acute intracerebral hemorrhage. Taken together, these findings indicated that Simvastatin exerted beneficial effects in patients with intracerebral hemorrhage by improving survival, vasospasm, brain edema, the amount of bleeding as well as limb coordination and walking.

\section{Acknowledgements}

This work was supported by the National Science foundation of China (no. 814020100354 to W.L.L.).

\section{References}

1. Sussman ES and Connolly ES Jr: Hemorrhagic transformation: A review of the rate of hemorrhage in the major clinical trials of acute ischemic stroke. Front Neurol 4: 69, 2013.

2. Kobayashi T, Tadokoro H, Odai T, Hibino T and Waki K: A delayed cerebral vasospasm with infarction is secondary to listeria monocytogenes meningitis: MRI and MRA are diagnostically useful. Intern Med 54: 2935-2938, 2015.

3. Lee WJ, Yeon JY, Jo KI, Kim JS and Hong SC: Reversible cerebral vasoconstriction syndrome and posterior reversible encephalopathy syndrome presenting with deep intracerebral hemorrhage in young women. J Cerebrovasc Endovasc Neurosurg 17: 239-245, 2015.

4. Kanamaru K, Suzuki H and Taki W: Cerebral infarction after aneurysmal subarachnoid hemorrhage. Acta Neurochir Suppl 121: 167-172, 2016.

5. Brown RJ, Epling BP, Staff I, Fortunato G, Grady JJ and McCullough LD: Polyuria and cerebral vasospasm after aneurysmal subarachnoid hemorrhage. BMC Neurol 15: 201, 2015.

6. Jenson AV, Rodriguez GJ, Alvarado LA, Cruz-Flores S and Maud A: Higher rate of intracerebral hemorrhage in hispanic patients with cerebral Cavernous Malformation. J Vasc Interv Neurol 8: 1-4, 2015.

7. Dabus G and Nogueira RG: Current options for the management of aneurysmal subarachnoid hemorrhage-induced cerebral vasospasm: A comprehensive review of the literature. Interv Neurol 2: 30-51, 2013

8. Sehba FA, Pluta RM and Macdonald RL: Brain injury after transient global cerebral ischemia and subarachnoid hemorrhage. Stroke Res Treat 2013: 827154, 2013.

9. Matsumoto $\mathrm{H}$ and Yoshida Y: Rapid progression of cerebral infarction after intraventricular hemorrhage in adult moyamoya disease. J Korean Neurosurg Soc 54: 411-414, 2013.

10. Medeiros de Bustos E and Moulin T; French Society of Intensive Care: Specific treatment for intracerebral hemorrhage. Experts' recommendations: Stroke management in the intensive care unit. Rev Neurol (Paris) 168: 522-526, 2012 (In French).

11. Ohwaki K, Yano E, Nagashima H, Nakagomi T and Tamura A: Impact of infection on length of intensive care unit stay after intracerebral hemorrhage. Neurocrit Care 8: 271-275, 2008.

12. Ma L, Shen X, Gao Y, Wu Q, Ji M, Luo C, Zhang M, Wang T, Chen $\mathrm{X}$ and Tao L: Blocking B7-1/CD28 pathway diminished long-range brain damage by regulating the immune and inflammatory responses in a mouse model of intracerebral hemorrhage. Neurochem Res 41: 1673-1683, 2016.

13. Boehme AK, Hays AN, Kicielinski KP, Arora K, Kapoor N, Lyerly MJ, Gadpaille A, Shiue H, Albright K, Miller D, et al: Systemic inflammatory response syndrome and outcomes in intracerebral hemorrhage. Neurocrit Care 25: 133-140, 2016.

14. Schuiling WJ, Dennesen PJ and Rinkel GJ: Extracerebral organ dysfunction in the acute stage after aneurysmal subarachnoid hemorrhage. Neurocrit care 3: 1-10, 2005. 
15. McGirt MJ, Mavropoulos JC, McGirt LY, Alexander MJ, Friedman AH, Laskowitz DT and Lynch JR: Leukocytosis as an independent risk factor for cerebral vasospasm following aneurysmal subarachnoid hemorrhage. J Neurosurg 98: 1222-1226, 2003.

16. Panichi V, Paoletti S, Mantuano E, Manca-Rizza G, Filippi C, Santi S, Taccola D, Donadio C, Tramonti G, Innocenti M, et al: In vivo and in vitro effects of simvastatin on inflammatory markers in pre-dialysis patients. Nephrol Dial Transplant 21: 337-344, 2006.

17. Montazerolghaem M, Ning Y, Engqvist H, Karlsson Ott M, Tenje M and Mestres G: Simvastatin and zinc synergistically enhance osteoblasts activity and decrease the acute response of inflammatory cells. J Mater Sci Mater Med 27: 23, 2016.

18. Encarnação IC, Xavier CC, Bobinski F, dos Santos AR, Corrêa M, de Freitas SF, Aragonez A, Goldfeder EM and Cordeiro MM Analysis of bone repair and inflammatory process caused by simvastatin combined with PLGA+HA+ $\beta$ TCP Scaffold. Implant Dent 25: 140-148, 2016.

19. Shahbazian H, Atrian A, Yazdanpanah L, Lashkarara GR and Zafar Mohtashami A: Anti-inflammatory effect of simvastatin in hemodialysis patients. Jundishapur J Nat Pharm Prod 10: e17962, 2015.

20. Bartolomé F, Muñoz U, Esteras N, Alquezar C, Collado A, Bermejo-Pareja F and Martín-Requero A: Simvastatin overcomes the resistance to serum withdrawal-induced apoptosis of lymphocytes from Alzheimer's disease patients. Cell Mol Life Sci 67: 4257-4268, 2010.

21. Pinchuk TV, Fedulaev YN, Khairetdinova GA, Denisova NN Chura OV and Logunova IY: Anti-inflammatory effects of simvastatin in patients with chronic heart failure. Bull Exp Biol Med 157: 552-554, 2014.

22. Lacerda L, Reddy JP, Liu D, Larson R, Li L, Masuda H, Brewer T, Debeb BG, Xu W, Hortobágyi GN, et al: Simvastatin radiosensitizes differentiated and stem-like breast cancer cell lines and is associated with improved local control in inflammatory breast cancer patients treated with postmastectomy radiation. Stem Cells Transl Med 3: 849-856, 2014.

23. Guan C, Liu W, Yue Y, Jin H, Wang X and Wang XJ: Inhibitory effect of $\beta$-elemene on human breast cancer cells. Int J Clin Exp Pathol 7: 3948-3956, 2014.

24. Kirmizis D, Papagianni A, Dogrammatzi F, Efstratiadis G and Memmos D: Anti-inflammatory effects of simvastatin in diabetic compared to non-diabetic patients on chronic hemodialysis. J Diabetes 5: 492-494, 2013.

25. Sugawara T, Ayer R, Jadhav V, Chen W, Tsubokawa T and Zhang JH: Simvastatin attenuation of cerebral vasospasm after subarachnoid hemorrhage in rats via increased phosphorylation of Akt and endothelial nitric oxide synthase. J Neurosci Res 86: 3635-3643, 2008.

26. McGirt MJ, Lynch JR, Parra A, Sheng H, Pearlstein RD, Laskowitz DT, Pelligrino DA and Warner DS: Simvastatin increases endothelial nitric oxide synthase and ameliorates cerebral vasospasm resulting from subarachnoid hemorrhage. Stroke 33: 2950-2956, 2002.

27. Teoh EJ, McGowan DR, Bradley KM, Belcher E, Black E, Moore A, Sykes A and Gleeson FV: 18F-FDG PET/CT assessment of histopathologically confirmed mediastinal lymph nodes in non-small cell lung cancer using a penalised likelihood reconstruction. Eur Radiol 26: 4098-4106, 2016.

28. Matsukawa H, Tanikawa R, Kamiyama H, Tsuboi T, Noda K, Ota N, Miyata S, Suzuki G, Takeda R and Tokuda S: Effects of Clot removal by meticulous irrigation and continuous low-dose intravenous nicardipine on symptomatic cerebral vasospasm in patients with aneurysmal subarachnoid hemorrhage treated by clipping. World Neurosurg 84: 1798-1803, 2015.

29. Filipova J, Rihova L, Vsianska P, Kufova Z, Kryukova E, Kryukov F and Hajek R: Flow cytometry in immunoglobulin light chain amyloidosis: Short review. Leuk Res, Jul 13, 2015 (Epub ahead of print).

30. Sreekrishnan A, Dearborn JL, Greer DM, Shi FD, Hwang DY, Leasure AC,Zhou SE, Gilmore EJ, Matouk CC, Petersen NH, et al: Intracerebral hemorrhage location and functional outcomes of patients: A systematic literature review and meta-analysis. Neurocrit Care 25: 384-391, 2016

31. Rannikmäe K, Woodfield R, Anderson CS, Charidimou A, Chiewvit P, Greenberg SM, Jeng JS, Meretoja A, Palm F, Putaala J, et al: Reliability of intracerebral hemorrhage classification systems: A systematic review. Int J Stroke 11: 626-636, 2016.
32. Pittella JE and da Silva Gusmão SN: Intracerebral hemorrhage due to cerebral amyloid angiopathy after head injury: Report of a case and review of the literature. Neuropathology 36: 566-572, 2016.

33. Karki K, Knight RA, Han Y, Yang D, Zhang J, Ledbetter KA, Chopp M and Seyfried DM: Simvastatin and atorvastatin improve neurological outcome after experimental intracerebral hemorrhage. Stroke 40: 3384-3389, 2009.

34. Shao H, Shen Y, Liu H, Dong G, Qiang J and Jing H: Simvastatin suppresses lung inflammatory response in a rat cardiopulmonary bypass model. Ann Thorac Surg 84: 2011-2018, 2007.

35. Wang KW, Cho CL, Chen HJ, Liang CL, Liliang PC, Tsai YD, Wang HK and Lu K: Molecular biomarker of inflammatory response is associated with rebleeding in spontaneous intracerebral hemorrhage. Eur Neurol 66: 322-327, 2011.

36. Chamnanvanakij S, Margraf LR, Burns D and Perlman JM: Apoptosis and white matter injury in preterm infants. Pediatr Dev Pathol 5: 184-189, 2002

37. Riggs AJ and Riggs JE: Epilepsy's role in the historical differentiation of religion, magic, and science. Epilepsia 46: 452-453, 2005.

38. Gao F, Guo Y, Zhang H, Wang S, Wang J, Wu JM, Chen Z and Ding MP: Anterior thalamic nucleus stimulation modulates regional cerebral metabolism: An FDG-MicroPET study in rats. Neurobiol Dis 34: 477-483, 2009

39. Guo FQ, Li XJ, Chen LY, Yang H, Dai HY, Wei YS, Huang YL, Yang YS, Sun HB, Xu YC and Yang ZL: Study of relationship between inflammatory response and apoptosis in perihematoma region in patients with intracerebral hemorrhage. Zhongguo Wei Zhong Bing Ji Jiu Yi Xue 18: 290-293, 2006 (In Chinese).

40. Loftspring MC, Johnson HL, Feng R, Johnson AJ and Clark JF: Unconjugated bilirubin contributes to early inflammation and edema after intracerebral hemorrhage. J Cereb Blood Flow Metab 31: 1133-1142, 2011.

41. Wang J: Preclinical and clinical research on inflammation after intracerebral hemorrhage. Prog Neurobiol 92: 463-477, 2010.

42. Liao W, Zhong J, Yu J, Xie J, Liu Y, Du L, Yang S, Liu P, Xu J, Wang J, et al: Therapeutic benefit of human umbilical cord derived mesenchymal stromal cells in intracerebral hemorrhage rat: Implications of anti-inflammation and angiogenesis. Cell Physiol Biochem 24: 307-316, 2009.

43. Lee ST, Chu K, Sinn DI, Jung KH, Kim EH, Kim SJ, Kim JM, KoSY,Kim Mand Roh JK: Erythropoietin reduces perihematomal inflammation and cell death with eNOS and STAT3 activations in experimental intracerebral hemorrhage. J Neurochem 96: 1728-1739, 2006.

44. Wu L and Chen G: Signaling pathway in cerebral vasospasm after subarachnoid hemorrhage: News update. Acta Neurochir Suppl 121: 161-165, 2016.

45. Diringer MN, Dhar R, Scalfani M, Zazulia AR, Chicoine M, Powers WJ and Derdeyn CP: Effect of high-dose simvastatin on cerebral blood flow and static autoregulation in subarachnoid hemorrhage. Neurocrit Care 25: 56-63, 2016.

46. McGirt MJ, Garces Ambrossi GL, Huang J and Tamargo RJ: Simvastatin for the prevention of symptomatic cerebral vasospasm following aneurysmal subarachnoid hemorrhage: A single-institution prospective cohort study. J Neurosurg 110: 968-974, 2009.

47. Kidwell CS, Chalela JA, Saver JL, Starkman S, Hill MD, Demchuk AM, Butman JA, Patronas N, Alger JR, Latour LL, et al: Comparison of MRI and CT for detection of acute intracerebral hemorrhage. JAMA 292: 1823-1830, 2004.

48. Roever L and Levine SR: Cerebral hemorrhage following thrombolytic therapy for stroke: Are neutrophils really neutral? Neurology 85: 1360-1361, 2015

49. Wang DN, Hou XW, Yang BW, Lin Y, Shi JP and Wang N: Quantity of cerebral microbleeds, antiplatelet therapy, and intracerebral hemorrhage outcomes: A systematic review and meta-analysis. J Stroke Cerebrovasc Dis 24: 2728-2737, 2015.

50. Zhou HX, Gao LH, Meng LL, Zhang YX, Wei ZF and Si DW: Preventive and therapeutic effect of simvastatin on secondary inflammatory damage of rats with cerebral hemorrhage. Asian Pac J Trop Med 10: 152-156, 2017.

51. Lin C, Zhao Y, Wan G, Zhu A and Wang H: Effects of simvastatin and taurine on delayed cerebral vasospasm following subarachnoid hemorrhage in rabbits. Exp Ther Med 11: 1355-1360, 2016. 